\title{
A SHORT PROOF THAT COMPACT QUASIDEVELOPABLE SPACES ARE METRIZABLE
}

\section{H. R. BENNETT}

\begin{abstract}
A new characterization of quasidevelopable spaces is given that allows an
\end{abstract} easier proof that compact quasidevelopable spaces are metrizable.

In $\left[\mathbf{B}_{1}, \mathbf{B}_{2}\right]$ it was shown that a compact quasidevelopable Hausdorff space is metrizable. In this note a new characterization of quasidevelopable spaces is given that allows a much easier proof. Let all spaces be at least Hausdorff spaces and let $N$ denote the set of natural numbers.

A space $X$ is quasidevelopable if there exists a sequence $G_{1}, G_{2}, \ldots$ (called the quasidevelopment ) of collections of open subsets of $X$ such that if $x$ is in an open set $U$, then there exists $n \in N$ such that $\varnothing \neq \operatorname{st}\left(x, G_{n}\right) \subset U$.

THEOREM 1. A space $(X, \tau)$ is quasidevelopable if and only if there exists a function $g: N \times X \rightarrow \tau(g$ is called q.d. function) such that

(i) if $x \in X$, then $\{g(n, x) \mid n \in N, g(n, x) \neq \varnothing\}$ is a nonincreasing local base at $x$,

(ii) if $x \in g(n, y)$, then $g(n, x) \neq \varnothing$, and

(iii) if $\{x\}$ is not open in $X$ and if $g\left(n, z_{n}\right)$ contains $x$ and $y_{n}$ for each $n \in N$ such that $g(n, x) \neq \varnothing$, then some subsequence of $\left\langle y_{n} \mid g(n, x) \neq \varnothing\right\rangle$ converges to $x$.

Proof. Let $G_{1}, G_{2}, \ldots$ be a quasidevelopment for $X$. If $x \in X$ and $x \in \cup G_{n}$, let $g^{\prime}(n, x)$ be any element of $G_{n}$ that contains $x$. Let

$$
g(n, x)=\cap\left\{g^{\prime}(i, x) \mid i \leqslant n, x \in \cup G_{i}\right\} .
$$

Clearly (i) and (ii) are satisfied. If $\{x\}$ is not open in $X$ and $x$ and $y_{n}$ are in $g\left(n, z_{n}\right)$, then $y_{n} \in \operatorname{st}\left(x, C_{n}\right)$ for all $n \in N$ such that $x \in \cup G_{n}$. Thus some subsequence of $\left\langle y_{n} \mid g(n, x) \neq \varnothing\right\rangle$ converges to $x$.

If $g: N \times X \rightarrow \tau$ is a q.d. function, let $G_{1}=\{\{x\} \mid\{x\}$ open in $X\}$ and $G_{n+1}=$ $\{g(n, x) \mid x \in X, g(n, x) \neq \varnothing\}$. It easily follows that $G_{1}, G_{2}, \ldots$ is a quasidevelopment for $X$.

This characterization should be compared with a similar characterization of Moore spaces in $[\mathbf{H}]$.

THEOREM 2. Let $M$ be an infinite subset of $N$ and $g$ a q.d. function on $X$. If $g\left(m, y_{m}\right) \supset g\left(m+1, y_{m+1}\right)$ for each $m \in M$ and the sequence $\left\langle y_{m} \mid m \in M\right\rangle$ converges to some $p \in \cap\left\{g\left(m, y_{m}\right) \mid m \in M\right\}$, then $\left\{g\left(m, y_{m}\right) \mid m \in M\right\}$ is a local base at $p$.

Received by the editors May 3, 1982 and, in revised form, May 28, 1982.

1980 Mathematics Subject Classification. Primary 54E35, 54E45.

Key words and phrases. Compact, quasidevelopable, metrizable. 
Proof. Let $n_{1}$ be the first natural number such that $g\left(n_{1}, p\right) \neq \varnothing$. Let $m_{1} \geqslant n_{1}$ be the first member of $M$ such that $y_{m_{1}} \in g\left(n_{1}, p\right)$. Then $g\left(n_{1}, y_{m_{1}}\right) \neq \varnothing$ and $g\left(n_{1}, y_{m_{1}}\right)$ $\supseteq g\left(m_{1}, y_{m_{1}}\right)$. Inductively let $n_{k}$ be the first natural number larger than $n_{k-1}$ such that $g\left(n_{k}, p\right) \neq \varnothing$. Let $m_{k}$ be the first natural number such that $m_{k} \geqslant n_{k}$ and $m_{k}>m_{k-1}$ such that $y_{m_{k}} \in g\left(n_{k}, p\right)$. Thus $g\left(n_{k}, y_{m_{k}}\right) \neq \varnothing$ and $g\left(n_{k}, y_{m_{k}}\right) \supseteq$ $g\left(m_{k}, y_{m_{k}}\right)$.

If $\left\{g\left(n_{k}, y_{m_{k}}\right) \mid k \in N\right\}$ is not a local base at $p$ then there is an open set $U$ containing $p$ such that for each $k \in N$ there exists $t_{k} \in g\left(n_{k}, y_{m_{k}}\right)$ such that $t_{k} \notin U$. But, by (iii) of Theorem 1, some subsequence of $\left\langle t_{k} \mid k \in N\right\rangle$ converges to $p$. From this contradiction it follows that $\left\{g\left(n_{k}, y_{m_{k}}\right) \mid k \in N\right\}$ is a local base at $p$. Since $g\left(n_{k}, y_{m_{k}}\right) \supset g\left(m_{k}, y_{m_{k}}\right)$, it follows that $\left\{g\left(m, y_{m}\right) \mid m \in M\right\}$ is a local base at $p$.

Recall that a perfect (= closed sets are $G_{\delta}$-sets) quasidevelopable space is developable $\left[\mathbf{B}_{\mathbf{2}}\right]$.

\section{THEOREM 3. A compact quasidevelopable space is metrizable.}

Proof. Let $X$ be a compact quasidevelopable space with q.d. function $g$ and let $D=\{x \mid\{x\}$ is open in $X\}$. Let $C$ be a closed set in $X-D$. Then $C$ is compact. If $x \in C$, let $n(x, 1)$ be the first natural number such that $g(n(x, 1), x) \neq \varnothing$. Let $H_{1}$ be a finite subcollection of $\{g(n(x, 1), x) \mid x \in C\}$ which covers $C$. Suppose $H_{1}, \ldots, H_{k-1}$ have been chosen such that each $H_{i}, 1<i \leqslant k-1$, is a finite open cover of $C$ and if $h \in H_{i+1}$, then there exists $h^{\prime} \in H_{i}$ such that $h^{\prime} \supset \operatorname{cl}(h)$ (= closure of $h$ in $\left.X\right)$. If $x \in C$, let $n(x, k)>k$ be the first natural number such that $\operatorname{cl}(g(n(x, k), x)) \subset$ $\cap\left\{h \in H_{k-1} \mid x \in h\right\}$. Let $H_{k}$ be a finite subcollection of $\{g(n(x, k), x) \mid x \in C\}$ which covers $C$.

Suppose there exists $q \in X-C$ such that $q \in \cup H_{k}$ for each $k \in N$. Then, using König's lemma [K], there is a sequence $\left\langle h_{k} \mid k \in N\right\rangle$ such that $h_{k} \in H_{k}, \operatorname{cl}\left(h_{k+1}\right) \subset$ $h_{k}$ and $q \in h_{k}$ for each $k \in N$. For each $k \in N, h_{k}=g\left(n_{k}, x_{k}\right)$ for some $x_{k} \in C$ where we have written $n_{k}=n\left(x_{k}, k\right)$. Since $x_{k} \in C$ and since $X$ is first-countable, the sequence $\left\langle x_{k} \mid k \in N\right\rangle$ has a subsequence which converges to some $p \in$ $\cap\left\{\operatorname{cl}\left(h_{k}\right) \mid k \in N\right\} \cap C$. Without loss of generality let $\left\langle x_{k} \mid k \in N\right\rangle$ converge to $p$. By Theorem 2, $\left\{g\left(n_{k}, x_{k}\right) \mid k \in N\right\}$ is a local base at $p$. Thus, for some $k \in N$, $g\left(n_{k}, x_{k}\right) \subset X-\{q\}$. From this contradiction it follows that $q \notin \cup H_{k}$ and, hence, $C=\cap\left\{\cup H_{i} \mid i \in N\right\}$. Thus $C$ is a $G_{\delta}$-set in $X$.

If $K$ is a closed subset of $X$ then $K=C \cup D^{\prime}$ where $D^{\prime} \subset D$ and $C \cap D=\varnothing$. Since $C \subset X-D, C=\bigcap\left\{O_{i} \mid i \in N\right\}$ where each $O_{i}$ is open in $X$. Then $K=$ $\cap\left\{O_{i} \cup D^{\prime} \mid i \in N\right\}$ and thus, $X$ is perfect. Thus $X$ is a compact developable space and, hence, metrizable $\left[\mathbf{B}_{2}\right]$.

\section{REFERENCES}

[B $\left.\mathbf{B}_{1}\right]$ H. R. Bennett, A note in the metrizability of M-spaces, Proc. Japan Acad. 45 (1969), 6-9.

$\left[\mathbf{B}_{2}\right] \ldots$, On quasi-developable spaces, General Topology Appl. 2 (1972), 49-55.

[H] R. W. Heath, Arcwise connectedness in semi-metric spaces, Pacific J. Math. 12 (1962), 1301-1319.

[K] K. Kunen, Set theory, An introduction to independence proofs, vol. 102, North-Holland, Amsterdam, 1980.

Department of Mathematics, TeXas Tech University, Lubbock, TeXas 79409 